\title{
非线性演化方程的对称性 和守恒律之间的关系
}

\author{
屠规彰秦孟兆 \\ (中国科学院计算中心)
}

自应用科学中的一个新概念一一孤立子（Soliton）提出以来,在物理学的许多领域中已发 现了众多的具有孤立子解的非线性演化方程 ${ }^{[1]}$. 研究表明, 这些演化方程具有一个引人注目 的特色, 即具有无穷多个守恒律. 如所周知,一个具有 Lagrangian 的系统, 守恒律常与系统的 不变变换群 (亦即对称性) 密切相关 (Noether 定理 ${ }^{[2]}$ ). 但此处不变变换系对 Lagrangian 而 言,而 Euler 方程的不变变换未必使其 Lagrangian 不变 ${ }^{[3]}$. 另一方面,在微分方程的相似解理 论 ${ }^{[4]}$ 中,已广泛发展了 Lie 的寻求微分方程不变群的系统方法, 因而能否直接从 Euler 方程的 不变群出发去构造相应的守恒律, 乃是一个很重要而又未解决的问题 ${ }^{[5]}$. 况且, Noether 定理 中所要求的不变变换允许 Lagrangian 的变化相差一个散度量, 因此寻求此种不变变换时除去 确定变换群的母元外, 还需确定与此散度量相应的未知向量, 这较之直接求 Euler 方程的不变 群更为困难.

Wadati ${ }^{[31}$ 曾就 $\mathrm{KdV}$ 方程 $u_{t}+u u_{x}+u_{x x x}=0$ 经相当复杂的推理证明了此方程的守恒 律与一类形如 $\eta=\frac{d}{d x} \mathscr{Y} f$ (算子 $\mathscr{Y}$ 的定义见后) 的不变群母元间的关系. Kumei ${ }^{[6]}$ 则就稍 为广泛些的方程 $u_{t}+a(u) u_{x}+u_{x \varepsilon x}=0$ 给出了同一关系式, 但他在证明中, 实际上假定了此 类方程具有充分多的无穷小不变变换 $f \rightarrow f+\varepsilon \phi$, 因而其论证是不完备的. 本文则就十分一 般的非线性演化方程建立了守恒律与对称性之间的明确的关系，而且变换群母元的形式不必 限于上述的 $\eta=\frac{d}{d x} \mathscr{Y} f$ 形式. 这一结果将有助于构造一大类具有无穷多个守恒律的非线性 演化方程.

给定一个变分问题 $\min \int f\left(x, u, u_{1}, \cdots, u_{n}\right) d x$, 其中 $u_{i} \equiv \frac{d^{i}}{d x^{i}} u$ 为末知函数 $u=u(x)$ 的各阶导数,其 Euler 方程为 $\mathscr{Y} f=0$, 其中算子 $\mathscr{Y}$ 定义为

$$
\mathscr{Y} \equiv \sum_{i>0}(-\mathscr{D})^{i} \partial_{i} \quad\left(\mathscr{D} \equiv \frac{d}{d x}, \partial_{i} \equiv \frac{\partial}{\partial u_{i}}\right)
$$

与算子 $\mathscr{Y}$ 相关,我们引人次之三类算子, 式中 $f=f\left(u, u_{1}, \cdots\right)$ 为 $u_{i}$ 的函数:

$$
\begin{gathered}
\mathscr{Y}_{k}=\sum_{i \geqslant k}\left(\begin{array}{l}
i \\
k
\end{array}\right)(-\mathscr{D})^{i-k} \partial_{i}, \\
\mathscr{V}^{\wedge}(f)=\sum_{i \geqslant 0}\left(\partial_{i} f\right) \mathscr{D}^{i} \quad \mathscr{U}(f)=\sum_{i \geqslant 0}\left(\mathscr{D}^{i} f\right) \partial_{i} .
\end{gathered}
$$

本文 1979 年 3 月 5 日收到. 
注意到 $\mathscr{Y}_{0}=\mathscr{Y}$, 又 $\mathscr{Y}_{k} \mathscr{D}^{i}=\mathscr{Y}_{k-i}(k \geqslant i), \mathscr{Y}_{b} \mathscr{D}^{i}=0(k<i)^{[7]}$.

定义 1 若 $g=g\left(x, u, u_{1}, \cdots, u_{n}\right)$ 可以写成 $g=\mathscr{Y} f$ 的形式,则称 $g$ 具有 Lagrangian 密度, 尤当此种 $g$ 为 $u_{i}$ 的常系数多项式且不含常数项 (即 $g(0)=0$ ) 时, 称 $g$ 为梯度多项 式.

定理 $\mathbf{A}^{*[8]}$ 对于 $g=g\left(x, u_{1}, u_{2}, \cdots, u_{n}\right), \partial_{n} g \neq 0$, 其次四个性质相互等价: (i) $g$ 具有 Lagrangian 密度 (亦即 $g=0$ 为某个变分问题的 Euler 方程); (ii) $\mathscr{V}(g)=\mathscr{V}^{*}(g)$, ( $\mathscr{V}^{*}$ 表示微分算子 $\mathscr{Y}$ 的形式共轭);（iii） $\mathscr{Y}_{i} g=(-1)^{i} \partial_{i} g,(i=0,1, \cdots, n)$; (iv) $n=$ $2 m$ 且 $\mathscr{Y}_{2 k-1} g=-\partial_{2 k-1} g(k=1,2, \cdots, m)$.

由多个变量情形的定理 A 可以证明: 形如

$$
u_{t}=H\left(u, u_{1}, \cdots, u_{n}\right)
$$

的非线性演化方程不可能是某个变分问题的 Euler 方程,但若对 (1) 式作变量代换 $u=w_{x}$, 则可转而考察方程

由定理 $A$ 不难推知

$$
w_{x t}=K\left(w, w_{1}, \cdots, w_{p}\right), \quad w_{i} \equiv \mathscr{D}^{i} w
$$

命题 1 为了方程 (1)'为某个变分问题的 Euler 微分方程,必须而且只须 $K(w)$ 具有 Lagrange 密度, 且若以 $L_{E}$ 记 $E \equiv K(w)-w_{x t}$ 的 Lagrange 密度,而以 $L_{K}$ 记 $K(w)$ 的 Lagrange 密度, 则

$$
L_{E}=L_{K}+\frac{1}{2} w_{\imath} w_{x}
$$

例如对 $\mathrm{KdV}$ 方程, $u_{t}=u u_{1}+u_{3}$, 相应的 $K=w_{1} w_{2}+w_{4}$, 易得 ${ }^{[7]} L_{K}=\left(3 w_{2}^{2}-w_{1}^{3}\right) / 6$ ， 从而由命题 $1, L_{E}=\omega_{1} \omega_{x} / 2+\left(3 w_{x x}^{2}-w_{x}^{3}\right) / 6$.

由定理 A 注意到 $\partial_{k} \mathscr{D}=\mathscr{D} \partial_{k}+\partial_{k-1}$ 不难推出

命题 2 若 $g(u)$ 为梯度多项式, 则 $g(w) \equiv \mathscr{D} g\left(w_{x}\right)$ 亦为梯度多项式.

由命题 $1 \cdot 2$ 可见,形如

$$
u_{t}=\mathscr{D} g(u)
$$

的非线性演化方程, 其中 $g=g\left(u, u_{1}, \cdots, u_{n}\right)$ 为梯度多项式, 在作代换 $u=w_{x}$ 后均可作 为某个变分问题的 Euler 方程.

由算子 $\mathscr{S}_{k}$ 的定义易得 ${ }^{[8]}$

$$
\mathscr{Y}_{s}(f g)=\sum_{p}(-1)^{p}\left(\begin{array}{c}
p+s \\
s
\end{array}\right)\left[\left(\mathscr{D}^{p} g\right) \mathscr{Y}_{p+s} f+\left(\mathscr{D}^{p} f\right) \mathscr{Y}_{p+s} g\right]
$$

特别

$$
\mathscr{Y}(f g)=\sum_{p}(-1)^{p}\left[\left(\mathscr{D}^{p} g\right) \mathscr{Y}_{p} t+\left(\mathscr{D}^{p} f\right) \mathscr{Y}_{p} g\right] .
$$

定义 2 设 $f, g$ 与 $h$ 均为 $u_{i}$ 的函数, 若有关系式 $f=g+\mathscr{D} h$, 则称 $f$ 与 $g$ 为 $\mathscr{D}$-等 价，并记作 $f \stackrel{\mathscr{D}}{=} g$.

例如由 Leibniz 公式知 $f \mathscr{D} g \stackrel{\mathscr{D}}{=}-g \mathscr{D} f$. 反复应用此式可得

$$
\mathscr{U}(g) f=\mathscr{Y}(f) g \stackrel{\mathscr{D}}{=}(\mathscr{Q} f) g,
$$

* 见屠规彰、泰孟兆，《Euler-Lagrange 方程的判别准则》一文(即将发表). 
引理 1 (i) $\mathscr{V}(f g)=f \mathscr{V}(g)+g \mathscr{V}(f)$; (ii) $\mathscr{D} \mathscr{V}(f)=\mathscr{V}(\mathscr{D} f)$; (iii) $\mathscr{D} g=\mathscr{V}(g) u_{1}$; (iv) $\mathscr{D}=\Sigma u_{i+1} \partial_{i} ;$ (v) $\mathscr{Y} f=0 \Leftrightarrow f \stackrel{\mathscr{D}}{=} 0$.

(i) (iv) 由定义显见，(v) 见文献 ${ }^{[7]}$.

由定理 A (iii) 及 (4)' 式, 易得

命题 3 若 $f$ 与 $g$ 均为梯度多项式, 则

$$
\mathscr{Y}(f \mathscr{D} g)=\mathscr{V}(f) \mathscr{D} g-\mathscr{V}(g) \mathscr{D} f,
$$

尤取 $f=u$, 由引理 1 的 (iii)并注意到 $\mathscr{V}(u)=1$ 即得

命题 4 若 $g$ 为梯度多项式, 则 $\mathscr{Y}(u \mathscr{D} g)=0$.

由引理 $1(v)$, 又注意到 $u_{1} g=\mathscr{D}(u g)-u \mathscr{D} g$ 可将上述命题改写成

命题 $4^{\prime}$ 若 $g$ 为梯度多项式, 则

$$
u \mathscr{D g} \stackrel{\mathscr{D}}{=} u_{1} \stackrel{\mathscr{D}}{=} 0 .
$$

今设函数 $f(u)=f\left(u, u_{1}, u_{2}, \cdots\right)$, 若 $u=u(x, \alpha)$ 与某参数 $\alpha$ 相关, 则由算子 $\mathscr{V}(f)$ 的定义易见

$$
\frac{d}{d \alpha} f(u)=\mathscr{V}(f) \stackrel{d u}{d \alpha},
$$

特别当 $u=u(x, t)$ 为演化方程 $u_{t}=H(u)$ 的解时, 有

$$
-\frac{\partial}{\partial t} f(u)=\mathscr{V}(f) H
$$

由 (5) 及 (6)' 式可见, 当 $u$ 为 $u_{t}=H$ 的解时

$$
\frac{\partial}{\partial t} f(u) \stackrel{\mathscr{D}}{=}(\mathscr{Q} f) H .
$$

对于方程 (1) 的解 $u$, 若存在 $f(u)$ 与 $g(u)$, 使得

$$
\frac{\partial}{\partial t} f(u)+\frac{\partial}{\partial x} g(u)=0,
$$

则称方程 (1) 具有守恒律 (8) 式, 这时称 $f(u)$ 为守恒密度. 由 (7) 式及引理 1 (v) 可见

命题 $5 f(u)$ 为方程 (1) 的守恒密度的充要条件是

$$
(\mathscr{Y} f) H \stackrel{\mathscr{D}}{=} 0 \text { 或 } \mathscr{Y}[(\mathscr{Y} f) H]=0 .
$$

换言之, 寻找方程 (1) 的守恒律, 归结为求得 $H(u)$ 的一个梯度多项式型的积分因子 $g$, 使乘 积 $g H$ 可表为对 $x$ 的全导数形式.

对于方程 (1), 设有无穷小变换

$$
u \rightarrow \mathrm{v}=u+\varepsilon \eta,
$$

其中 $\varepsilon$ 为无穷小参数, $\eta=\eta\left(u, u_{1}, u_{i}, \cdots\right)$, 使得当 $u$ 为 (1) 的解时 $\mathrm{v}_{t}-H(v)=O\left(\varepsilon^{2}\right)$, 则称 $(10)$ 式为无穷小不变变换, 并称算子 $\mathscr{U}(\eta)=\Sigma\left(\mathscr{D}^{i} \eta\right) \partial_{i}$ 为此变换的母元 (下面为简 单见, 即称 $\eta$ 为母元).

定理 $1 \eta=\eta\left(u, u_{1}, \cdots\right)$ 为方程 (1) 的一个无穷小不变变换的母元的充要条件为

$$
\mathscr{V}(\eta) H-\mathscr{V}(H) \eta=0 .
$$

证 记 $\Phi(u)=u_{t}-H(u)$; 由 (6) 及 (6)' 式并注意到

$$
\Psi \cdot u+\varepsilon \eta)=\Phi(u)+\varepsilon\left(\frac{d \Phi\left(u^{\prime}\right)}{d \varepsilon}\right)_{\varepsilon=0}+O\left(\varepsilon^{2}\right)
$$


即可推证.

引理 2 设 $g=g\left(u, u_{1}, u_{2}, \cdots\right.$ ) 不含常数项 (即 $g(0)=0$ ), 则 $\mathscr{D} g=0 \Longrightarrow g=0$. (显然).

定理 2 设 $\eta=\eta\left(u, u_{1}, u_{2}, \cdots, u_{n}\right)$ 为演化方程

$$
u_{i}=\mathscr{D} g \quad(g \text { - 梯度多项式 })
$$

的无穷小不变变换的母元, 则

(i) $\eta=\mathscr{D} \mathscr{Y} f$ 当且仅当 $f$ 为同一方程 (3) 的守恒密度;

(ii) 若 $\eta$ 非 $\mathscr{D} \mathscr{P} f$ 的形式,则 $u \eta$ 为 (3) 式的守恒密度.

证 记 $H=\mathscr{D} g$. (i) $f$ 为(3)的守恒密度 $\Leftrightarrow$ (命题 5) $\mathscr{Y}((\mathscr{Y} f) \mathscr{D} g$ ) $=0 \Leftrightarrow($ 命题 3) $\mathscr{Y}(\mathscr{Q} f) \mathscr{D} g-\mathscr{V}(g) \mathscr{D} \mathscr{Q} f=0 \Leftrightarrow(引 1$ (ii)及引 2) $\mathscr{V}(\mathscr{D} \mathscr{Q} f) \mathscr{D} g-\mathscr{V}(\mathscr{D} g) \mathscr{D} \mathscr{Y} f=$ 0 , 此即 $\mathscr{V}(\eta) H-\mathscr{V}(H) \eta=0$, 故 $\Leftrightarrow$ (定理 1) $\eta$ 为 (3) 的无穷小不变变换的母元. (ii) $(u \eta)_{t}=$ (由(6)式) $\mathscr{V}(u \eta) H=$ (由引 $\left.1(\mathrm{i})\right)[\eta \mathscr{V}(u)+u \mathscr{V}(\eta)] H=\eta H+u \mathscr{V}(\eta) H$. 今 $H=\mathscr{D} g$, 故由命题 4, $\mathscr{Y}(u H)=0$, 从而

$$
-\eta H=\eta[\mathscr{S}(u H)-H]=\eta\left[\sum_{i>0}(-\mathscr{D})^{i} u \partial_{i} H\right] \stackrel{\mathscr{D}}{=} \sum_{i>0}\left(\mathscr{D}^{i} \eta\right) u \partial_{i} H=u \mathscr{V}(H) \eta,
$$

代入前一式即可见 $(u \eta)_{t} \stackrel{\mathscr{D}}{=} u[\mathscr{Y}(\eta) H-\mathscr{V}(H) \eta]=0$ (定理 1), 此即 $u \eta$ 为 (3) 的守恒密 度(此处顺便提及, 在 (ii) 中我们假设了 $\eta$ 非 $\mathscr{D} \mathscr{Y} f$ 形式, 此因当 $\eta=\mathscr{D} \mathscr{Y} f$ 时由命题 4', $u \eta \stackrel{D}{=} 0$ (对任意的 $u$ )，故所得 $u \eta$ 为平凡的守恒密度).

致谢：作者对冯康教授的热诚指导和鼓励,在此表示哀心感谢.

\section{考文朝}

[1] Scott, A. C. et al., IEEE proc., 61(1973),1443-1483.

[2] 刘文森, 山西大学学报, 1978,1:80-90.

[3] Lutzky, M., J. Phys. (A), 11 (1978), 249-258.

[4] Bluman, G. W. \& Cole, J. D., Similarity Methods for Differential Equations, Spring.Verlag, New York, 1974.

[5] Wadati, M., Studies in Appl. Math., 59 (1978), 153-186.

[6] Kumei, 8., J. Math. Phys., 18 (1977), 256-264.

[ 7 ] Kruskal, M. D. et al., J. Math. Phys., 11 (1970), 952-960.

[8] Atherton, R. W. \& Homsy, G. M., Studies in Appl. Math., 54 (1975), 31-60. 


\title{
RELATIONSHIP BETWEEN SYMMETRIES AND CONSERVATION LAWS OF NONLINEAR EVOLUTION EQUATIONS
}

\author{
Tu Gui-zhang (屠规彰) and Qin Mon-zhao (秦孟兆)
}

\section{Abstract}

In this paper we establish a relationship between the conservation law and symmetry for a general class of nonlinear evolution equations.

Theorem. For a nonlinear evoluton equation

$$
u_{t}=\mathscr{D} g \text {, }
$$

where $u=u(x, t), \mathscr{D}=\frac{d}{d x}, g=g\left(u, u_{1}, u_{2}, \cdots\right)$ is a gradient polynomial of $u_{i} \equiv \mathscr{D}^{i} u$ (i. e., the urdinary differential equation $g=0$ is an Euler equation of a certain variational problem), if $u \rightarrow u+\varepsilon \eta$ is an infinitesimal invariant transformation of equation (*), where $\varepsilon$ is an infinitesimal parameter, and $\eta=\eta\left(u, u_{1}, \ldots\right)$ is the function of $u_{i}$, then

(i) $\eta=\mathscr{D} \mathscr{Y} f$ if and only if $f$ is a conservation density of the equation (*), where the operator $\mathscr{Y}=\Sigma(-\mathscr{D})^{i} \cdot \frac{\partial}{\partial u_{i}}$;

(ii) If $\eta$ does not take the form of $\mathscr{D} \mathscr{Y} f$, then $u \eta$ is a conservation density.

This result would be helpful to the construction of a wide class of nonlinear evolution equations which possess an infinite number of conservation laws. 